PAPER

\title{
Brain metabolic decreases related to the dose of the ApoE e4 allele in Alzheimer's disease
}

\author{
L Mosconi, B Nacmias, S Sorbi, M T R De Cristofaro, M Fayazz, A Tedde, L Bracco, K Herholz, \\ A Pupi
}

J Neurol Neurosurg Psychiatry 2004;75:370-376

See end of article for authors' affiliations .....................

Correspondence to: Professor A Pupi Department of Clinical Pathophysiology, Nuclear Medicine Unit, University of Florence, viale Morgagni 85, 50134 Florence, Italy; a.pupi@dfc.unifi.it

Received 14 July 2003 Revised 21 July 2003

\begin{abstract}
Objectives: Declines in brain glucose metabolism have been described early in Alzheimer's disease (AD), and there is evidence that a genetic predisposition to $A D$ contributes to accelerate this process. The epsilon4 (e4) allele of the apolipoprotein E (ApoE) gene has been implicated as a major risk factor in this process. The aim of this FDG-PET study was to assess the ApoE e4 dose related effect on regional cerebral glucose metabolism (METglc) in clinical AD patients, with statistical voxel based methods.

Methods: Eighty six consecutive mild to moderate AD patients included in the Network for Efficiency and Standardisation of Dementia Diagnosis database underwent FDG-PET scans at rest. PCR was used to determine the ApoE genotype. Patients were grouped as e4 non-carriers $(n=46), e 3 / e 4(n=27)$ and e4/ e4 $(n=13)$ carriers. A voxel-based mapping program was used to compare each $A D$ subgroup with a database of 35 sex and age matched controls ( $p<0.001$, corrected for cluster extent) and also to compare between the subgroups $(p<0.001$, uncorrected).

Results: No difference was found as to age at examination, age at onset, sex, disease duration, educational level, or severity of dementia between $A D$ subgroups. Compared with controls, all $A D$ subgroups had equivalent METglc reductions in the precuneus, posterior cingulate, parietotemporal, and frontal regions. Direct comparisons between $A D$ subgroups indicated that patients with at least one e4 allele had METglc reductions within additional associative and limbic areas compared with e4 noncarriers.

Conclusions: The present FDG-PET study showed different metabolic phenotypes related to the ApoE genotype in clinical AD patients, as revealed with voxel based statistical methods. The results suggest a generalised disorder in e4 carriers impairing metabolism globally, in addition to the more localised changes typical of $A D$ patients.
\end{abstract}

up SPECT study showed reduced cerebral perfusion in the parietal and occipital cortices of e4 homozygous patients compared with e4 heterozygotes and non-carriers. ${ }^{15}{ }^{16}$ Conversely, the three studies that examined the gene dosing effect provided no evidence for a disrupting role of the e4 allele on brain functional parameters. ${ }^{17-19}$

In contrast, only one FDG-PET study has been performed so far with a voxel based analysis. ${ }^{20}$ Compared with the ROI technique, voxel based methods for anatomical image normalisation and statistical parametric mapping (SPM) allow assessment of all possible significant changes in the whole brain and at the single voxel level. ${ }^{21}{ }^{22}$ Using SPM, Hirono et al showed that carriers of the e4/e4 genotype have metabolic decreases in the medial temporal lobe and increases in the parietotemporal cortices compared with e3/ e3 carriers, and that this is related to the age at disease onset. ${ }^{20}$ However, this effect was observed for early onset patients only, and the gene dosage effect was not examined.

The present FDG-PET study was aimed to assess whether $\mathrm{AD}$ patients present with different metabolic phenotypes depending on their ApoE genotype. A voxel based analysis using the Statistical Parametric Mapping (SPM'99) program

\footnotetext{
Abbreviations: $A C C$, anterior cingulate cortex; $A D, A$ lzheimer's disease; ApoE, apolipoprotein E; BA, Brodmann area; FOV, field of view; METglc, glucose metabolism; MRI, magnetic resonance imaging; NEST-DD, Network for Efficiency and Standardisation of Dementia Diagnosis; PET, positron emission tomography; ROI, regions of interest; SPM, statistical parametric mapping; STL, superior temporal lobe
} 
was performed to assess the impact of the inherited dose of the e4 allele on regional cerebral glucose metabolism (METglc) in both early and late onset AD patients.

\section{MATERIALS AND METHODS AD patients}

Eighty-six consecutive mild AD patients were included in the study. Written informed consent was obtained from all patients, in accordance with institutional guidelines. All patients were diagnosed according to the criteria of the National Institute for Neurological Disorders and Stroke and of the Alzheimer's disease and Related Disorders Association. ${ }^{23}$ Subjects were included with or without a family history of $\mathrm{AD}$ (defined as at least one first degree relative with a clinical diagnosis of $\mathrm{AD}$ ). The study procedures included examinations by neurologists and psychiatrists, routine laboratory tests, neuropsychological examinations, EEG, magnetic resonance imaging (MRI), and positron emission tomography (PET) scans, and blood samples for genetic analysis. Patients were excluded in cases of: (a) complications of other medical illnesses possibly causing cognitive impairment, such as thyroid disease, vitamin deficiencies, and malignant disease; $(b)$ mental disease, substance abuse, or significant neurological antecedents (brain trauma, tumours, epilepsy, vascular dementia, other dementing illnesses, and inflammatory disease); or (c) evidence of focal brain lesions on MRI.

Subjects were recruited in the context of the Network for Efficiency and Standardisation of Dementia Diagnosis (NEST-DD) research project (www.nest-dd.org) funded by the EEC, and were studied for cerebral glucose metabolism by PET between November 2000 and November 2002 at our institute. Patients were enrolled in this study based on their genetic characterisation. DNA was obtained from blood samples to determine the ApoE genotypes, by using standard PCR, as described by Sorbi et al. ${ }^{24}$ Three AD subgroups were decided upon: (a) 46 ApoE4 non-carriers (8 with the e2/e3 genotype and 38 with the e3/e3 genotype); (b) 27 ApoE4 heterozygotes (e3/e4 genotype); and (c) 13 ApoE4 homozygotes (e4/e4 genotype).

The characteristics of the patients under study are reported in table 1 . We used analysis of variance to test differences in demographic and clinical data between AD subgroups. The Scheffé method was applied in post hoc analyses. The level of significance was set at $\mathrm{p}<0.05$.

\section{Controls}

Thirty-five healthy and unmedicated sex and age matched subjects (19M, 16F; mean age 69.3 (5.6) years, range 64-81 years; education: 10.3 (3.2) years; MMSE 26 or higher), included in the NEST-DD database, were used as controls. With their informed consent and approval by local ethics committees, they had been studied with PET under the same

Table 1 Demographic and clinical characteristics of AD patients carrying the different ApoE genotypes

\begin{tabular}{llll}
\hline \multicolumn{4}{c}{ AD subgroups } \\
\cline { 2 - 4 } & Non-e4 & e3/e4 & e4/e4 \\
\hline $\mathrm{n}$ & 46 & 27 & 13 \\
Age (years)* & $73(13)$ & $76(9)$ & $72(7)$ \\
Age at onset (years) & $67(10)$ & $70(7)$ & $67(10)$ \\
MMSE scores* & $21.8(3.5)$ & $23.3(3.6)$ & $23.5(3.1)$ \\
Educational level (years)* & $7.8(4.8)$ & $6.2(3.1)$ & $8.6(5.3)$ \\
Length of illness (months)* & $50(23)$ & $48(21)$ & $59(33)$ \\
Gender (M/F) & $16 / 30$ & $8 / 19$ & $4 / 9$ \\
\hline \multirow{2}{*}{ *Values expressed as mean (SD). } & & \\
\hline
\end{tabular}

conditions and using the same scanning protocol at three collaborating laboratories, the Cologne, Liege, and Dresden centres, with PET scanners that differed with respect to field of view (FOV) and spatial resolution. ECAT EXACT, ECAT 951, and ECAT EXACT HR+ scanners were used, with an axial FOV of 162, 108, and $155 \mathrm{~mm}$, an in plane FWHM of 6, 8.5, and $3.6 \mathrm{~mm}$ and a slice thickness of $3.375,3.37$, and $2.46 \mathrm{~mm}$, respectively. Studies were performed on subjects in a resting state with eyes closed and ears unplugged after intravenous injection of 110 to $370 \mathrm{MBq} 18 \mathrm{~F}-2$-fluoro-deoxyD-glucose (FDG). Required minimum time interval between injection and scan start was $30 \mathrm{~min}$. On average, scans were started 42 (19) minutes after injection. Required minimum scan duration was 10 minutes, and mean scan duration was 19 (3) minutes. Images were reconstructed using filtered back projection including correction for attenuation (measured by transmission scan) and scatter using standard software as supplied by scanner manufacturers. Further details can be found in Herholz et al. ${ }^{8}$ All subjects underwent clinical and neuropsychological examinations to exclude organic brain disease and cognitive impairment.

PET

The detailed FDG-PET scanning procedure employed was as described by Bartenstein et al. ${ }^{25}$ Briefly, patients were injected with a dose of $370 \mathrm{MBq}\left[{ }^{18} \mathrm{~F}\right]$ fluoro-2-deoxy-Dglucose $\left({ }^{18} \mathrm{~F}-\mathrm{FDG}\right)$, in a resting state, with eyes closed and ears unplugged, in a dimly lighted room with minimal background noise. Thirty minutes after FDG administration, a scan lasting 20 minutes was acquired, using a GE Advance PET device. A polycarbonate head holder was used to reduce head movement during the scan. Emission scans were acquired in two dimensional mode with an axial FOV of $15 \mathrm{~cm}$ and a planar FOV of $50 \times 50 \mathrm{~cm}$. Images were reconstructed by filtered back projection. Attenuation correction was performed according to Chang's method. ${ }^{26}$ Thirtyfive PET slices were acquired using a $256 \times 256$ matrix with a pixel dimension of $2.14 \times 2.14 \mathrm{~mm}$ and the slice thickness was $4.25 \mathrm{~mm}$.

\section{Image transformation}

A MS-DOS version of NEUROSTAT (kindly provided by the Department of Internal Medicine, University of Michigan, Ann Arbor, MI, USA) running on a Windows Me system was used to realign PET images relative to the anterior-posterior commissural line (AC-PC) and to normalise them to the FDG-PET template provided by NEUROSTAT to match the standard stereotactic Talairach and Tournoux space. ${ }^{27}$ Normalisation to the Talairach space was done using a nine parameter linear affine transformation and a non-linear warping deformation. ${ }^{22}$

Thereafter PET images were converted into Analyze format using ImageJ software (http://rsb.info.nih.gov/ij/). Voxel by voxel statistical analysis was performed with SPM'99 (Wellcome Functional Imaging Laboratory, London, UK) implemented in MATLAB, (version 5.3). Images were smoothed with an isotropic gaussian filter $(12 \mathrm{~mm}$ full width at half maximum) to account for individual variability in structure-function relation and to enhance signal to noise ratio. ${ }^{21}$ Individual global counts were normalised by proportional scaling. The grey matter threshold was set to 0.8 of the mean and global calculation was obtained with respect to the mean voxel value.

\section{Statistical analysis}

Comparisons between controls and $A D$ subgroups Each $\mathrm{AD}$ genotypic subgroup (ApoE4 non-carriers $v$ e3/e4 genotype $v$ e4/e4 genotype) was compared with the database of healthy controls. Differences between groups were 
estimated according to the general linear model using linear $t$ contrasts. The significance threshold was set at $\mathrm{p}<0.001$, corrected for cluster extent. The resulting statistical parametric maps (SPMs) were converted to Z scores. Brain areas identified through the SPMs were then identified and labelled according to the Talairach and Tournoux space $^{27}$ by using the Talairach Daemon Database 2002 (http://ric.uthscsa.edu/projects/talairachdaemon.html). ${ }^{28}$

\section{Comparisons between $A D$ subgroups}

The pooled data were assessed with the $F$ statistic to identify the brain regions that significantly differed between the three AD genotypic subgroups. Results were considered significant at $\mathrm{p}<0.001$, uncorrected for multiple comparisons. Brain areas showing significant differences between groups were then identified through the SPMs and labelled as described in the previous paragraph.

As the $F$ statistic is non-directional, post hoc tests are required to assess which group mean differs from the others. The post hoc Scheffé test was used to compare METglc data between AD subgroups, using STATISTICA [version 4.5; StatSoft Inc, 1993]. Direct group mean comparisons were performed on the normalised METglc volumes extracted from the brain areas associated with significant differences between AD subgroups. METglc data were extracted from spherical regions of $10 \mathrm{~mm}$ diameter, centred at each local maximum of statistical significance. The left and right sides of each brain area were separately taken into account. The critical value for statistical significance was set at $\mathrm{p}<0.05$.

\section{RESULTS}

\section{Patient demographics}

There were no significant differences between AD subgroups in gender distribution, age at examination, age at disease
Table 3 Peak voxel localisation and significance of the brain regions characterised by significant metabolic differences between $A D$ genotypic subgroups (e4 noncarriers, e3/e4, and e4/e4). Results reaching significance at $p<0.001$ and at $p<0.005$ are presented in separate sections.

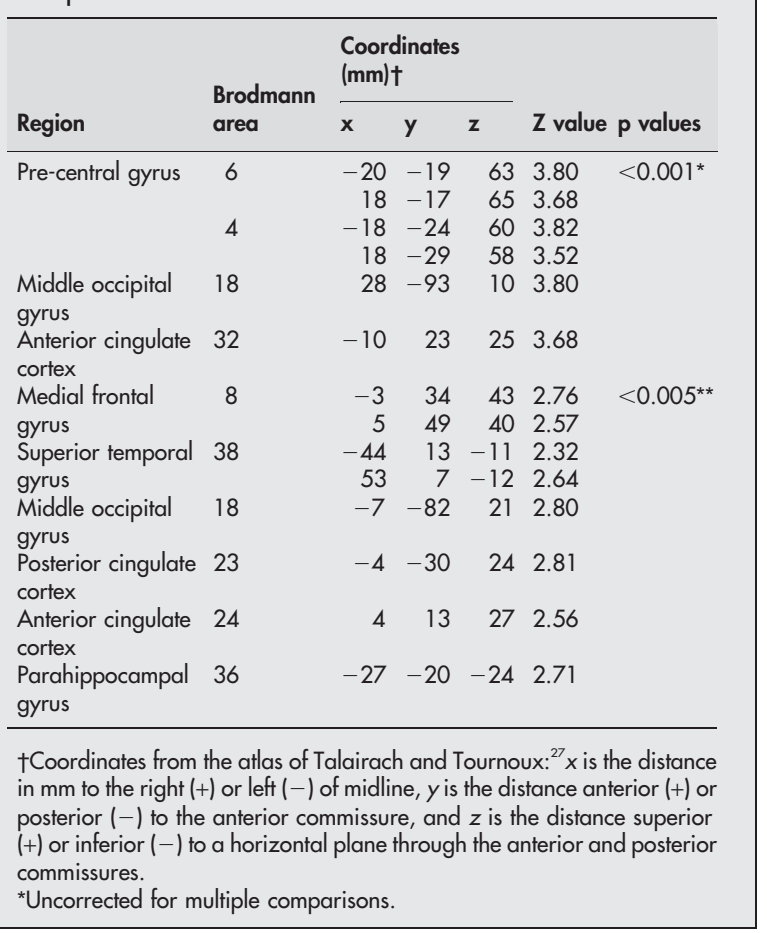

onset, disease duration, years of educational level, or severity of disease (table 1).

Table 2 Peak voxel localisation and significance of the brain regions characterised by reduced metabolism in the e4 non-carriers (non-e4), e3/e4, and e4/e4 AD patients compared with controls

\begin{tabular}{|c|c|c|c|c|c|c|c|}
\hline \multirow[b]{2}{*}{ Comparison } & \multirow[b]{2}{*}{$\mathrm{Ke}$} & \multirow[b]{2}{*}{ Region } & \multirow{2}{*}{$\begin{array}{l}\text { Brodmann } \\
\text { area }\end{array}$} & \multicolumn{3}{|c|}{ Coordinates $(\mathrm{mm}) \dagger$} & \multirow[b]{2}{*}{ Z value } \\
\hline & & & & $\mathbf{x}$ & $y$ & $\mathbf{z}$ & \\
\hline \multirow[t]{10}{*}{ Non-e4 } & 8637 & Inferior parietal lobule & 40 & -58 & -47 & 38 & 7.11 \\
\hline & & Fusiform gyrus & 20 & -50 & -30 & -19 & 5.49 \\
\hline & 1108 & Superior frontal gyrus & 8 & -26 & 43 & 40 & 6.85 \\
\hline & & Middle frontal gyrus & 8 & -46 & 22 & 43 & 5.41 \\
\hline & & Superior frontal gyrus & 9 & -18 & 54 & 32 & 5.41 \\
\hline & 3185 & Inferior parietal lobule & 40 & 58 & -44 & 44 & 6.06 \\
\hline & & Supramarginal gyrus & 40 & 59 & -59 & 32 & 5.40 \\
\hline & 192 & Inferior temporal gyrus & 20 & 58 & -40 & -17 & 5.45 \\
\hline & 621 & Precuneus & 7 & -2 & -57 & 35 & 5.44 \\
\hline & & Cingulate gyrus & 31 & 4 & -55 & 28 & 5.30 \\
\hline \multirow[t]{7}{*}{ e3/e4 } & 62 & Superior frontal gyrus & 8 & -26 & 43 & 40 & 6.15 \\
\hline & 1498 & Inferior parietal lobule & 40 & -55 & -55 & 38 & 5.22 \\
\hline & & Supramarginal gyrus & 40 & -56 & -52 & 28 & 5.92 \\
\hline & 810 & Supramarginal gyrus & 40 & 60 & -55 & 36 & 5.84 \\
\hline & 201 & Middle frontal gyrus & 8 & 30 & 39 & 44 & 5.29 \\
\hline & 482 & Precuneus & 7 & 4 & -66 & 38 & 5.90 \\
\hline & & Cingulate gyrus & 31 & -3 & -60 & 28 & 5.30 \\
\hline \multirow{8}{*}{ e4/e4 } & 1028 & Inferior parietal lobule & 40 & -46 & -60 & 42 & 6.22 \\
\hline & & Precuneus & 7 & -5 & -66 & 38 & 5.37 \\
\hline & & Cingulate gyrus & 23 & -3 & -57 & 18 & 5.26 \\
\hline & 1184 & Inferior parietal lobule & 39 & 50 & -66 & 40 & 6.23 \\
\hline & & Angular gyrus & 39 & 55 & -65 & 31 & 6.06 \\
\hline & 415 & Inferior temporal gyrus & 20 & 60 & -49 & -14 & 5.54 \\
\hline & 90 & Superior frontal gyrus & 8 & -26 & 41 & 42 & 5.29 \\
\hline & 88 & Middle frontal gyrus & 8 & 32 & 35 & 44 & 5.54 \\
\hline
\end{tabular}

†Coordinates from the atlas of Talairach and Tournoux: ${ }^{27} x$ is the distance in $\mathrm{mm}$ to the right $(+)$ or left $(-)$ of midline, $y$ is the distance anterior $(+)$ or posterior $(-)$ to the anterior commissure, and $z$ is the distance superior $(+)$ or inferior $(-)$ to a horizontal plane through the anterior and posterior commissures. ${ }^{*} \mathrm{P}<0.001$, corrected for cluster extent $(\mathrm{Ke})$. 


\section{FDG-PET data}

Comparisons between controls and AD subgroups All AD subgroups presented similar topographical patterns of METglc reductions compared with controls. Significant METglc reductions were found bilaterally in the frontal lobe, comprising the superior and middle frontal gyrus (Brodmann area (BA) 8, 9); the parietal lobe, including the inferior parietal lobule and the supramarginal gyrus (BA 39, 40); the inferior temporal gyrus (BA 20); the posterior cingulate gyrus (BA 31); and the precuneus (BA 7). The corresponding SPM results are listed in table 2 and displayed in fig 1 .

\section{Comparisons between AD subgroups}

Significant differences were found between $\mathrm{AD}$ genotypic subgroups (fig 2A). At the cortical level, group differences were found in the pre-central frontal gyrus (BA 6), bilaterally, and in the right middle occipital lobe (BA 18). Within the limbic system, the left anterior cingulate cortex (ACC) (BA 24) was involved. Anatomical localisation, Talairach coordinates, and statistical significance for each area are reported in table 3. Direct comparisons between the three $\mathrm{AD}$ subgroups showed that both the e3/e4 and e4/e4 carriers had lower METglc within the ACC and the occipital lobe compared with the e4 non-carriers, whereas the e4/e4 carriers had lower METglc in the pre-central frontal cortex, bilaterally, compared with both e4 non-carriers and e3/e4 carriers (fig 2C).

In addition, by resetting the threshold at an exploratory probability level of $\mathrm{p}<0.005$ (fig 2B), bilateral METglc differences emerged in the medial frontal gyrus (BA 8), the occipital lobe (BA 18), the ACC (BA 32/24), and the superior temporal lobe (STL) (BA 38). Unilateral group differences, present only in the left hemisphere, were found in the posterior cingulate cortex (PCC, BA 23) and the parahippocampal gyrus (PHG, BA 36). Direct comparisons within these brain regions showed that e4/e4 patients have lower METglc in the frontal cortex, bilaterally, and in the left PCC and PHG, compared with the e3/e4 carriers and the e4 non-carriers both. Conversely, both the e4/e4 and the e3/e4 carriers have lower METglc within the STL, ACC, and occipital lobe, bilaterally, compared with the e4 noncarriers (fig 2C). Although these additional findings have only an exploratory validity, they show that the metabolic differences between $\mathrm{AD}$ subgroups in relation to ApoE4 dose involve brain areas adjacent to those typically hypometabolic in $\mathrm{AD}$ in comparison with normal controls (fig 3).

\section{DISCUSSION}

The present FDG-PET study shows that different metabolic phenotypes can be detected with a voxel based statistical approach in a population of mild $\mathrm{AD}$ patients, related to the inherited dose of the ApoE e4 allele.

This work provides two main findings. Compared with the control subjects, all AD genotypic subgroups had equivalent patterns of METglc reductions in the precuneus, posterior cingulate, parietotemporal, and frontal regions. In contrast, $\mathrm{AD}$ subgroups presented significant metabolic differences in the cortical and limbic areas adjacent to those hypometabolic in $\mathrm{AD}$ patients, compared with controls (fig 3). Within those areas, the e4/e4 carriers had greater METglc reductions compared with the e 4 non-carriers, whereas the e3/e4 carriers had variable reductions depending on the brain area considered.

When compared with normal controls, all AD subgroups presented with similar patterns of METglc reductions within the precuneus, posterior cingulate, parietotemporal, and frontal association cortex. These regions are typically affected in $\mathrm{AD}$ and the rate of metabolic impairment was found to be strongly related to the degree of cognitive impairment. ${ }^{8}$ As showed by Herholz et al, ${ }^{8}$ all regions related to AD severity are already affected in very mild $\mathrm{AD}$, suggesting that all vulnerable areas are already affected to a similar degree at disease onset. Consistently, the present study showed that $\mathrm{AD}$ subgroups comparable for both disease severity (as assessed through MMSE scores) and mean age at onset have equivalent spatial distribution of metabolic decreases within these areas.

These results are consistent with previous ROI studies on $\mathrm{AD}$ patients that reported no difference between genotypic subgroups within the precuneus, posterior cingulate, parietotemporal, and frontal areas. ${ }^{17-19}$ Furthermore, Hirono et $a^{20}$ provided evidence that the hypometabolism within the parietotemporal and medial temporal areas varies between early onset e3/e3 and e4/e4 carriers, but that these differences are lost for late onset patients.

Together with previous research, the present results suggest that at the onset of $\mathrm{AD}$ cognitive symptoms, possible METglc differences between the ApoE genotype groups are no longer detectable within these mainly hypometabolic areas in $\mathrm{AD}$. In a 2 year follow up study, Lehtovirta et al ${ }^{16}$ reported that the e4/e4 carriers had greater rCBF reductions in those areas compared with e3/e4 carriers and e4 noncarriers, thus providing evidence that parieto-occipital rCBF differences can still be detected in clinical $\mathrm{AD}$ patients by longitudinal assessments.
Non E4
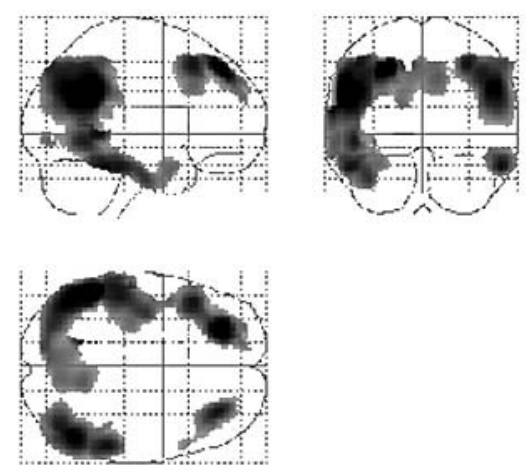

E3/E4
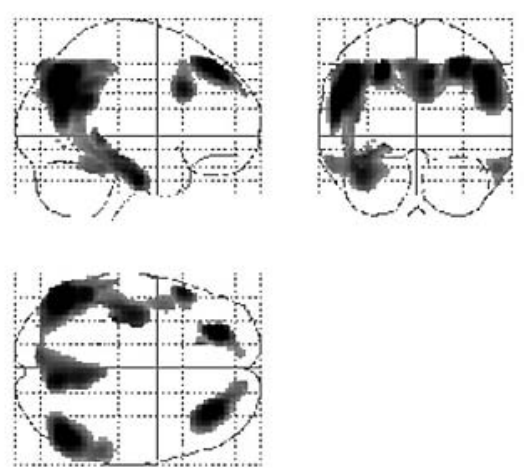

E4/E4
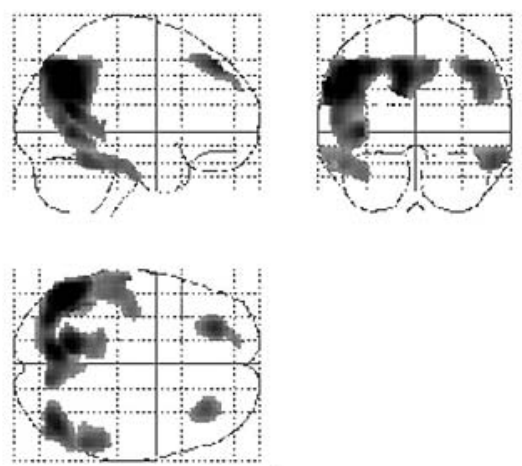

Figure 1 Statistical parametric maps (SPMs) showing areas of significantly lower METglc in e4 non-carriers (non-e4, left panel), e3/e4 (middle), and e4/e4 carriers AD patients (right panel), compared with control subjects ( $<0.001$, corrected for cluster extent). Results (black) are displayed as SPM projections in the three orthogonal right lateral (left), posterior (middle), and superior (below left) views. 
A

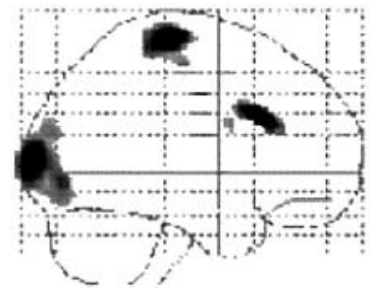

B

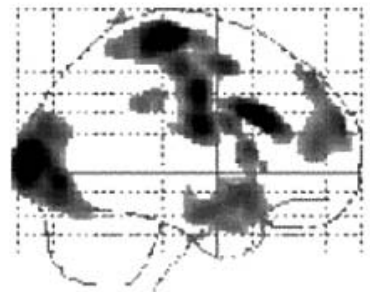

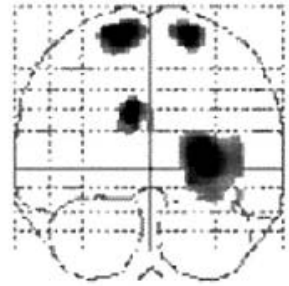
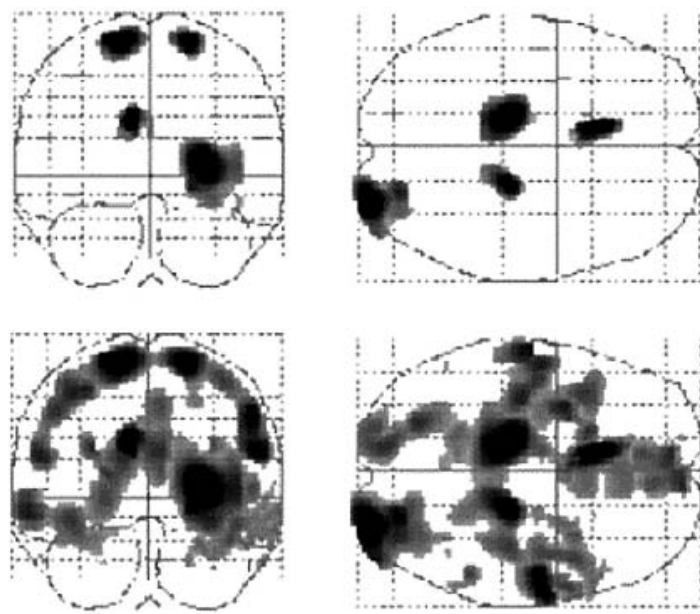

C

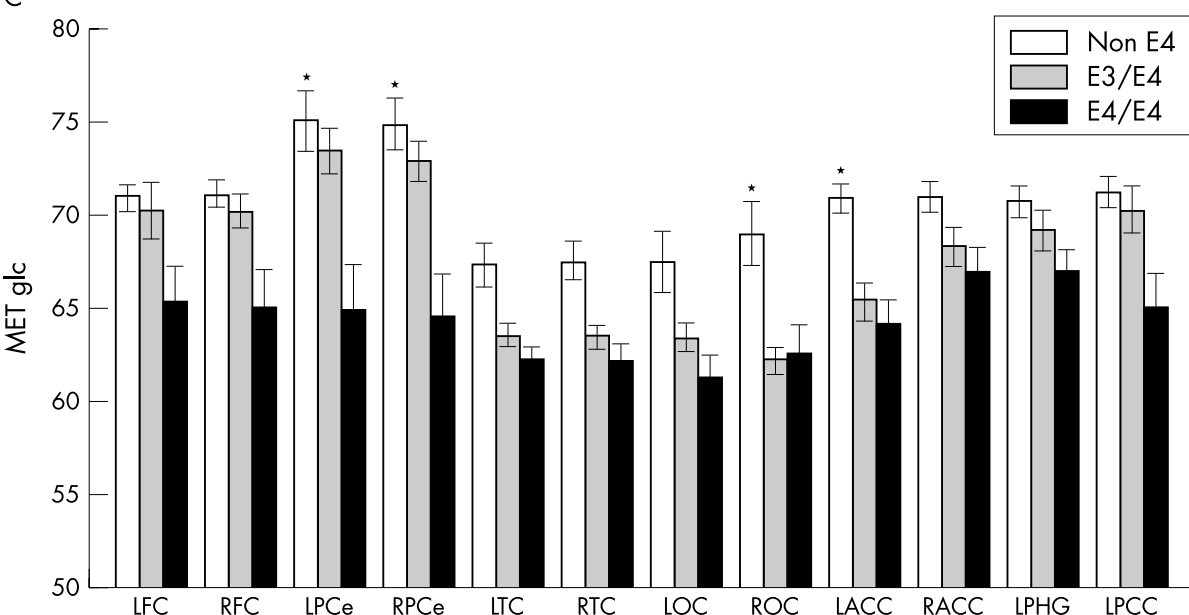

Figure 2 Metabolic differences in AD patients. Statistical parametric maps (SPMs) showing areas of regional cerebral metabolic rate of glucose (METglc) difference between ApoE4 non-carriers (non-e4), e3/e4 and e4/ e4 carriers, reported at $p<0.001$ (A) and $p<0.005(B)$, uncorrected. Results (black) are displayed as SPM

projections in the three orthogonal right lateral (left), posterior (middle) and superior (right) views. (C) METglc data extracted from the brain areas listed in table 3 and corresponding to the SPMs displayed in (B). METglc was normalised to a mean voxel value of $50 \mu \mathrm{mol} / 100 \mathrm{~g} / \mathrm{min}^{38}{ }^{38} \mathrm{Pe}$, precentral cortex; FC, frontal cortex; $O C$ occipital cortex; TC, temporal cortex; ACC, anterior cingulate cortex; PCC, posterior cingulate cortex; $\mathrm{PHG}$, parahippocampal gyrus; $L$, left; $R$, right. Error bars represent SEM. Group differences significant at $p<0.001$ are marked with asterisks $\left({ }^{*}\right)$.
Figure 3 Statistical parametric maps (SPMs) showing (A) areas of significant METglc decrease in AD patients compared with control subjects ( $p<0.001$, corrected for cluster extent); (B) areas of significant METglc differences between ApoE4 noncarriers, e3/e4 and e4/e4 allele carriers ( $p<0.005$, uncorrected); (C) SPMs resulting from $(A)$ (red) and (B) (green) are superimposed onto the right lateral, posterior, and superior surfaces of a spatially standardised volume rendered MRI image.
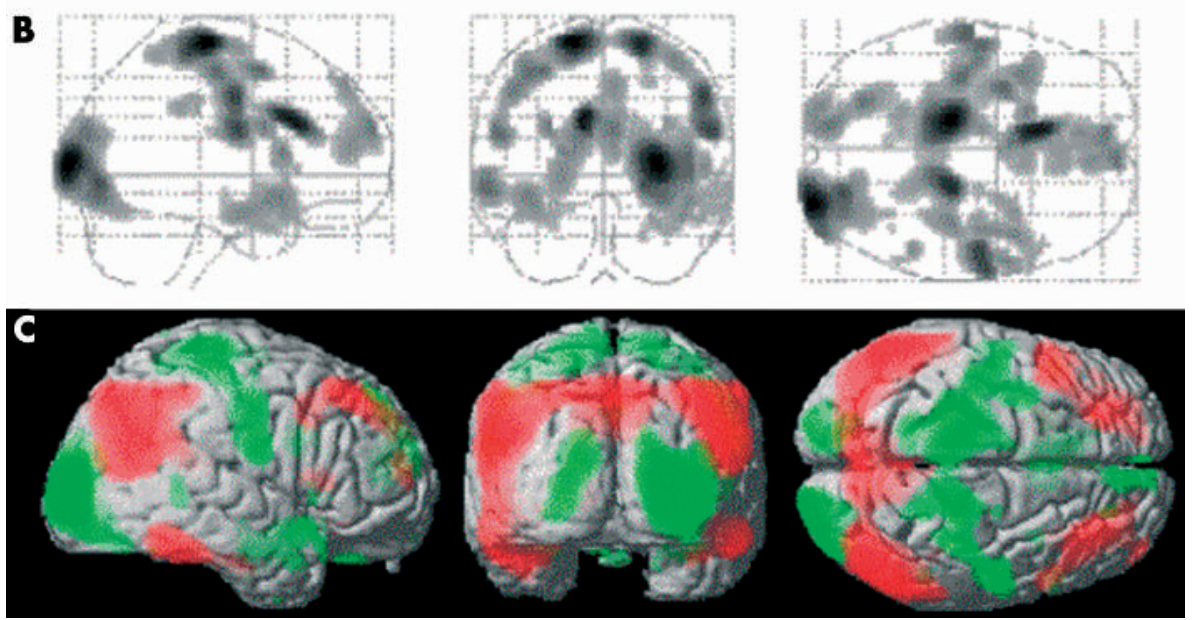
The comparison between the $\mathrm{AD}$ subgroups showed that both e3/e4 and e4/e4 carriers have lower METglc within the pre-central areas compared with e4 non-carriers, whereas within the occipital and ACC areas the e4/e4 carriers have lower METglc compared with both e4 non-carriers and e3/e4 carriers. In addition, by resetting the threshold at a slightly lower probability level $(\mathrm{p}<0.005)$, lower METglc in the e4/e4 compared with the non-carriers was found in several cortical and limbic areas surrounding the regions referred to above and filling the gaps between these and the typically hypometabolic regions in $\mathrm{AD}$ (fig 3 ). These additional regions include the medial frontal gyrus, middle occipital lobe, ACC, superior temporal lobe, posterior cingulate cortex, and parahippocampal gyrus. Although these findings have only an exploratory validity, they show that ApoE related phenotypic differences are evident within the brain areas adjacent to those typically hypometabolic in $\mathrm{AD}$ (fig 3).

It should be noted that the pre-central and occipital regions are usually metabolically spared in $\mathrm{AD}$ compared with normal controls. ${ }^{8}$ Indeed, the present findings are derived from the comparison between genetically different $\mathrm{AD}$ patients rather than with the controls, and therefore suggest that these regions express a METglc impairment in an e4 dose related fashion that is still below the detection threshold compared with normal controls. These findings could reflect a relative preservation of FDG uptake in the e4 non-carriers and, in turn, a relatively diffuse disruption in the e 4 carriers, consistent with genetic data showing a protective effect of the e 2 and e 3 alleles, and an e4 related pathological function in $\mathrm{AD} .{ }^{10}$ The present results are compatible with the hypothesis that the e4 allele plays a disruptive role in METglc. Indeed, FDG-PET studies on cognitively normal elderly showed that, compared with non-carriers of the e4 allele, e4 heterozygotes and homozygotes had abnormally low METglc in the same brain areas as clinical AD patients. Additionally, the METglc decline continued over time and aggravated in the e4 carriers. ${ }^{5}{ }^{13}{ }^{14}$ Concurring with these findings, the present study shows that increasing ApoE4 load enhances the $\mathrm{AD}$ related METglc disruption, with a subtle proportional involvement still undetectable compared with normal controls. This effect could be better investigated through a voxel by voxel analysis rather than with the ROI approach, because of the small size and the unexpected anatomical position of the brain regions involved. Future studies with different samples of $\mathrm{AD}$ patients are needed to validate these exploratory findings.

Furthermore, we found that the influence of the e4 allele dose on METglc is regionally selective. This effect has not been explored so far because the phenotypic differences occur within brain areas that were not examined in previous ROI studies. ${ }^{16-19}$ Indeed, the present study shows that at least one copy of the e4 allele is related to METglc reductions within the ACC, STL, and occipital lobe, whereas a double e4 allele dose is associated with lower METglc in the frontal cortex, PCC, and PHG. The mechanisms by which the different polymorphisms of the ApoE affect cerebral functioning are currently under investigation. ${ }^{1}$ Future studies are needed to evaluate the biological factors through which the different genotypes are implicated in the pattern of regionally specific disruption.

Lastly, the present findings provide evidence for an e4 related METglc decline in the anterior cingulate cortex (ACC). This finding is consistent with recent neuroimaging reports that have implicated the ACC in AD onset, ${ }^{29}{ }^{30}$ mainly because of its participation in the retrieval of episodic memories and in attentive processes, ${ }^{31-33}$ although independently of the ApoE genotype. Although there is evidence of a decline in cognitive abilities related to the e4 allele, ${ }^{34-36}$ further studies are needed to assess whether this effect could be associated with an ACC METglc reduction. Moreover, the parahippocampal gyrus, including the entorhinal cortex, presented a METglc reduction related to the ApoE genotype. This result is consistent with the findings by De Leon et al, ${ }^{7}$ who showed how lower METglc levels in the entorhinal cortex of normal elderly could predict conversion to mild cognitive impairment, with the e4/e4 carriers having the greater baseline declines.

In the present study, a correction for cortical atrophy was not performed. Nonetheless, most studies that have performed voxel based atrophy correction of resting glucose metabolism have reported a relative independence of METglc reduction from brain atrophy. ${ }^{37}$ Moreover, additional information could be provided by quantitative rather than relative METglc measurements.

Future studies are necessary to investigate whether the combination of genetic data and neuroimaging assessment could help to outline possible target groups for preventive procedures, and to design rational strategies for the treatment of $\mathrm{AD}$.

\section{ACKNOWLEDGEMENTS}

This study was conducted by the Network for Efficiency and Standardisation of Dementia Diagnosis with support from the European Commission (Framework V; European Union grants QLK-6-CT-1999-02178 and QLK-6-CT-1999-02112). Work at Florence was also supported by the Ministero dell'Università e della Ricerca Scientifica e Tecnologica, by Telethon Italia Fondazione ONLUS (grant no E.0980), and by Regione Toscana, Progetto Ministero della Sanita', "Diagnosi Tempestiva e Differenziale della Malattia di Alzheimer. Uso delle Tecnologie avanzate". Work at CRC Liege was also supported by grants from FNRS and FMRE, Belgium. The authors gratefully acknowledge Professors E Salmon and V Holthoff for kindly providing part of the database of healthy controls and Dr S Minoshima for allowing the use of NEUROSTAT software. We would like to thank $\operatorname{Dr} M$ Matteini for the help in patient management during scans, Drs E Cellini and S Bagnoli for their contribution in genetic assessments, and Professor A R Formiconi and $\mathrm{Mr} \mathrm{G}$ Comis for their assistance in image analysis.

\section{Authors' affiliations}

L Mosconi, M T R De Cristofaro, M Fayazz, A Pupi, Department of Clinical Pathophysiology, Nuclear Medicine Unit, University of Florence, Italy

B Nacmias, S Sorbi, A Tedde, L Bracco, Department of Neurological and Psychiatric Sciences, University of Florence, Italy K Herholz, Neurological Clinic and Max-Planck-Institute for Neurological Research, University of Cologne, Germany

Competing interest: none declared

\section{REFERENCES}

1 St George-Hyslop PH. Molecular genetics of Alzheimer's Disease. Biol Psychiatry 2001;47:183-99.

2 Mirra SS, Heyman A, McKeel D, et al. The Consortium to Establish a Registry for Alzheimer's Disease (CERAD). Part II. Standardization of the neuropathologic assessment of Alzheimer's disease. Neurology 1991;41:479-86.

3 Poirier J, Delisle MC, Quirion R, et al. Apolipoprotein E4 allele as a predictor of cholinergic deficits and treatment outcome in Alzheimer disease. Proc Natl Acad Sci USA 1995;92:12260-4.

4 Nobili F, Koulibaly M, Vitali P, et al. Brain perfusion follow-up in Alzheimer's patients during treatment with acetylcholinesterase inhibitors. J Nucl Med 2002;43:983-90.

5 Small GW, Mazziotta JC, Collins MT, et al. Apolipoprotein E type 4 allele and cerebral glucose metabolism in relatives at risk for familial Alzheimer's disease. JAMA 1995;273:942-7.

6 Reiman EM, Caselli RJ, Yun LS, et al. Preclinical evidence of Alzheimer's disease in persons homozygous for the epsilon 4 allele for apolipoprotein $E$. N Engl J Med 1996;334:752-8.

7 de Leon MJ, Convit A, Wolf OT, et al. Prediction of cognitive decline in normal elderly subjects with 2-[(18)F]fluoro-deoxy-D-glucose/positron-emission tomography (FDG/PET). Proc Natl Acad Sci USA 2001;98:10966-71.

8 Herholz K, Salmon E, Perani D, et al. Discrimination between Alzheimer dementia and controls by automated analysis of multicenter FDG PET. Neurolmage 2002;17:302-16. 
9 Farrer LA, Cupples LA, Haines JL, et al. Effects of age, sex, and ethnicity on the association between apolipoprotein $E$ genotype and Alzheimer disease. A meta-analysis. APOE and Alzheimer Disease Meta Analysis Consortium. JAMA 1997;278:1349-56.

10 Roses AD. Apolipoprotein E and Alzheimer's disease. The tip of the susceptibility iceberg. Ann NY Acad Sci 1998;855:738-43.

11 Corder EH, Saunders AM, Strittmatter WJ, et al. Gene dose of alipoprotein E type 4 allele and the risk of Alzheimer's disease in late onset families. Science 1993;261:921-3.

12 Poirier J, Davignon J, Bouthillier D, et al. Apolipoprotein E polymorphism and Alzheimer's disease. Lancet 1993;342:697-9.

13 Small GW, Ercoli LM, Silverman DH, et al. Cerebral metabolic and cognitive decline in persons at genetic risk for Alzheimer's disease. Proc Natl Acad Sci USA 2000;97:6037-42.

14 Reiman EM, Caselli RJ, Chen K, et al. Declining brain activity in cognitively normal apolipoprotein E epsilon 4 heterozygotes: A foundation for using positron emission tomography to efficiently test treatments to prevent Alzheimer's disease. Proc Natl Acad Sci USA 2001;98:3334-9.

15 Lehtovirta M, Soininen H, Laakso MP, et al. SPECT and MRI analysis in Alzheimer's disease: relation to apolipoprotein E epsilon 4 allele. J Neurol Neurosurg Psychiatry 1996;60:644-9.

16 Lehtovirta M, Kuikka J, Helisalmi S, et al. Longitudinal SPECT study in Alzheimer's disease: relation to apolipoprotein E polymorphism. J Neurol Neurosurg Psychiatry 1998;64:742-6.

17 Corder EH, Jelic V, Basun H, et al. No difference in cerebral glucose metabolism in patients with Alzheimer's disease and differing apoliprotein $\mathrm{E}$ genotypes. Arch Neurol 1997:54:273-7.

18 Hirono N, Mori E, Yasuda M, et al. Lack of association of apoliprotein E epsilon4 allele dose with cerebral glucose metabolism in Alzheimer's disease. Alzheimer Dis Assoc Disord 1998;12:362-7.

19 van Dyck CH, Gelernter J, MacAvoy MG, et al. Absence of an apoliprotein E epsilon4 allele is associated with increased parietal regional cerebral blood flow asymmetry in Alzheimer's disease. Arch Neurol 1998;55:1460-6.

20 Hirono N, Hashimoto M, Yasuda M, et al. The effect of APOE E4 allele on cerebral glucose metabolism in $A D$ is a function of age at onset. Neurology 2002; 58:743-50.

21 Friston KJ, Frith CD, Liddle PF, et al. Comparing functional (PET) images: the assessment of significant change. J Cereb Blood Flow Metab 1991;11:690-9.

22 Minoshima S, Koeppe RA, Frey KA, et al. Anatomical standardization: linear scaling and non linear warping of functional brain images. J Nucl Med 1994:35:1528-37.

23 McKhann G, Drachman D, Folstein M, et al. Clinical diagnosis of Alzheimer's disease: report of NINCDS/ADRDA Work Group under the auspices of
Department of Health and Human Services Task Force on Alzheimer's disease. Neurology 1984;34:939-44.

24 Sorbi S, Nacmias B, Forleo P, et al. Alzheimer's disease and apolipoprotein E in Italy. Ann NY Acad Sci 1996:777:260-5.

25 Bartenstein P, Asenbaum S, Catafau A, et al. European Association of Nuclear Medicine procedure guidelines for brain imaging using [(18)F]FDG. Eur J Nucl Med Mol Imaging 2002;29:43-8.

26 Chang LT. A method for attenuation correction in radionuclide computed tomography. IEEE Trans Nud Sci 1978;25:638-43.

27 Talairach J, Tournoux P. Co-planar stereotaxic atlas of the human brain: 3-dimensional proportional system-an approach to cerebral imaging. New York: Thieme Medical Publishers, 1988.

28 Lancaster JL, Woldorff MG, Parsons LM, et al. Automated Talairach Atlas labels for functional brain mapping. Human Brain Mapp 2000;10:120-31

29 Johnson KA, Jones BL, Holman JA, et al. Preclinical prediction of Alzheimer's disease using SPECT. Neurology 1998;50:1563-71.

30 Matsuda H, Kitayama N, Ohnishi T, et al. Longitudinal evaluation of both morphologic and functional changes in the same individuals with Alzheimer's disease. J Nucl Med, 2002:43:304-11.

31 Chetelat G, Baron JC. Early diagnosis of alzheimer's disease: contribution of structural neuroimaging. Neuroimage 2003;18:525-41.

32 Corbetta M, Miezin FM, Dobmeyer S, et al. Selective and divided attention during visual discriminations of shape, color, and speed: functional anatomy by positron emission tomography. J Neurosci 1991;11:2383-402.

33 Levine B, Cabeza R, Mclntosh AR, et al. Functional reorganisation of memory after traumatic brain injury: a study with $\mathrm{H}(2)(15) 0$ positron emission tomography. J Neurol Neurosurg Psychiatry 2002;73:173-81.

34 Helkala EL, Koivisto K, Hanninen T, et al. Memory functions in human subjects with different apolipoprotein $\mathrm{E}$ phenotypes during a 3-year population-based follow-up study. Neurosci Lett 1996;204:177-80.

35 Craft S, Teri L, Edland SD, et al. Accelerated decline in apoliprotein E-epsilon 4 homozygotes with Alzheimer's disease. Neurology 1998;51:149-53.

36 Caselli RJ, Graff-Radford NR, Reiman EM, et al. Preclinical memory decline in cognitively normal apolipoprotein E-epsilon4 homozygotes. Neurology 1999:53:201-7.

37 Ibanez V, Pietrini P, Alexander GE, et al. Regional glucose metabolic abnormalities are not the result of atrophy in Alzheimer's disease. Neurology 1998;50:1585-93.

38 Mazziotta JC, Phelps ME. Principles and Applications for the brain and heart. In: Phelps ME, Mazziotta JC, Schelbert H, eds. Positron emission tomography and autoradiography. New York: Raven Press, 1986:493-79. 